\title{
B-cell-specific Moloney murine leukemia virus integration site I: potential stratification factor and therapeutic target for epithelial ovarian cancer
}

This article was published in the following Dove Press journal:

OncoTargets and Therapy

22 August 2016

Number of times this article has been viewed

\author{
Qianying Zhaol,* \\ Ting Gui ${ }^{1, *}$ \\ Qiuhong Qian ${ }^{1,2}$ \\ Lei $\mathrm{Li}^{\prime}$ \\ Keng Shen' \\ 'Department of Obstetrics and \\ Gynecology, Peking Union Medical \\ College Hospital, Chinese Academy \\ of Medical Sciences \& Peking Union \\ Medical College, Beijing, ${ }^{2}$ Department \\ of Obstetrics and Gynecology, Qilu \\ Hospital of Shandong University, \\ Shandong, People's Republic of China \\ *These authors contributed equally \\ to this work
}

\begin{abstract}
Epithelial ovarian cancer, a vexing challenge for clinical management, still lacks biomarkers for early diagnosis, precise stratification, and prognostic evaluation of patients. B-cell-specific Moloney murine leukemia virus integration site 1 (BMI1), a member of the polycomb group of proteins, engages in diverse cellular processes, including proliferation, differentiation, senescence, and stem cell renewal. In addition, BMI1, as a cancer stem-cell marker, participates in tumorigenesis through various pathways. Rewardingly, recent studies have also revealed a relationship between BMI1 expression and the clinical grade/stage, therapy response, and survival outcome in a majority of human malignancies, including epithelial ovarian cancer. Therefore, BMI1 might serve as a potential stratification factor and treatment target for epithelial ovarian cancer, pending evidence from further investigations.
\end{abstract}

Keywords: B-cell-specific Moloney murine leukemia virus integration site 1 (BMI1), epithelial ovarian cancer (EOC), molecular marker, treatment target, cancer stem-cell, tumor heterogeneity

\section{Introduction}

Epithelial ovarian cancer (EOC) is a major lethal malignancy in the female genital system. The standard treatment consists of cytoreductive surgery, followed by frontline platinum/taxol chemotherapy. Although, $\sim 80 \%$ of patients initially respond to the treatment, a majority of them subsequently develop drug-resistant relapse and eventually succumb to the fatal disease. ${ }^{1}$ Given this scenario, there is an urgent need to understand better the etiology of this devastating disease, to develop more sensitive diagnostic methods and more precise stratification models for individualized treatment modalities.

B-cell-specific Moloney murine leukemia virus integration site 1 (BMII), initially identified as a $M y c$-cooperating oncogene, has a ring finger at the amino-terminus, which is required for the generation of murine lymphoma. ${ }^{2}$ Human BMII gene localizes on the short arm of chromosome 10 (10p11.23), and the encoded protein is ubiquitously expressed in almost all tissues, with high expression levels in the brain, esophagus, salivary gland, thymus, kidney, lungs, gonads, placenta, blood, and bone marrow. ${ }^{3}$ BMI1 is reported to play an important role in cell proliferation, immortalization, and senescence. ${ }^{4}$ An increasing number of studies demonstrate that BMI1 is upregulated in a variety of human malignancies, including nasopharyngeal, head and neck, liver, prostate, colorectal, breast, endometrial, cervical, and ovarian cancers. Moreover, the abnormal expression of BMI1 has also been proven to be significantly correlated with 
the clinical grade/stage of the disease, indicating the potential for BMI1 to serve as a therapeutic target and prognostic predictor of numerous cancers. ${ }^{4-14}$

Herein, we summarize the recent progress in understanding the biological functions and oncogenic role of BMI1. In particular, we focus on how BMI1 promotes the malignant phenotypes of EOC, and the associated advances in clinical application.

\section{Biological function of BMI I}

BMI1 protein, a polycomb group ( $\mathrm{PcG}$ ) family member, is one component of the polycomb repressive complex 1 , which catalyzes lysine 119 (K119) mono-ubiquitination of histone H2A (H2AK119Ub1). H2AK119Ub1, a repressive histone mark, is thought to contribute to gene silencing through the induction of chromatin compaction and inhibition of transcriptional elongation. ${ }^{15-17}$

BMI1 has a broad impact on diverse cellular processes, such as proliferation, differentiation, senescence, and contributes to embryonic and stem cell self-renewal programs. ${ }^{18}$ To date, many polycomb repressive complex 1 target genes have been identified including homeobox (HOX) genes and $p 16^{I N K 4 a}$, whose promoters contain interactive elements binding directly to BMI1. ${ }^{13}$ BMI1 controls the cell cycle by regulating the tumor suppressor proteins $\mathrm{p} 16^{\mathrm{INK} 4 \mathrm{a}}$ and $\mathrm{p} 14^{\mathrm{ARF}}$, and promotes cell proliferation by suppressing the $\mathrm{p} 16^{\mathrm{INK} 4 \mathrm{a}} /$ retinoblastoma and/or the $\mathrm{p} 14^{\mathrm{ARF}} / \mathrm{MDM} 2 / \mathrm{p} 53$ pathways. ${ }^{19}$ BMI1 was initially shown to regulate hematopoiesis and differentiation of lymphocytes and to be involved in cerebral development. ${ }^{20-22}$ BMI1 plays a crucial role in tissue homeostasis by maintaining self-renewal of hematopoietic, neural, prostate, intestinal, lung epithelial, and bronchioalveolar stem cells. ${ }^{4,21-23}$ BMI1 has also been reported to bypass senescence and immortalize cells by inducing the telomerase activity in adult stem cells. ${ }^{18}$ However, BMI1 fails to induce telomerase in human fibroblasts. ${ }^{4}$ Moreover, BMI1 is involved in inducing epithelial mesenchymal transition and is necessary for the optimal proliferation of CD8-positive T-cells. ${ }^{24,25}$ BMI1 is also pivotal for normal embryogenesis and cell identity: Pethe et al observed that BMI1 was upregulated when human embryonic stem cells differentiated into endoderm and ectoderm, while mesoderm differentiation was characterized by downregulation of all PcG transcripts including BMI1. ${ }^{26}$

\section{BMII in tumorigenesis: cancer stem cell and tumor heterogeneity}

At the germinal center of tumor evolution are cancer stem cells (CSCs), a stem cell-resembling population of cancer cells among the heterogeneous mix of cells constituting a tumor, which possesses the capacity of self-renewal and differentiation potential. ${ }^{27}$ The CSCs are posited to be responsible not only for tumor initiation but also for the generation of distant metastases. ${ }^{28}$ In addition, there is evidence indicating that CSCs, present within many tumor types, are spared by traditional cancer therapies, which typically target the rapidly dividing tumor cells, leading to relapse after therapy. 5,7,9,29 A long-standing goal of researchers is to establish a framework for understanding how many and which tumor cells must be eliminated for successful treatment.

Delightedly, a growing number of genetic and epigenetic expression profiling studies have revealed that the molecular traits, underlying human solid tumors in patients diagnosed with early-stage carcinomas of various origins, could predict a marked propensity toward metastatic dissemination, highly aggressive clinical behavior, and high probability of poor survival outcome. ${ }^{30}$ The ability of CSCs to induce cancer recurrence has been attributed to the activation of different molecules including BMI1.6,31 And emerging studies have conferred the important function of BMI1 as a biomarker of CSCs. ${ }^{32}$ Accumulating evidence has confirmed the oncogenic activation of BMI1 in diverse human malignancies. ${ }^{4-14,25,33}$ In addition, numerous recent studies have explored the function of BMI1 and the related pathways that serve as regulator in both stem and cancer cells.

BMI1 is required for self-renewal and maintenance of stem cells. ${ }^{34}$ Disruption of BMI1 signaling has been linked to the activation of the hedgehog pathway in some cancers, for example, medulloblastoma. ${ }^{35}$ BMI1 regulates multiple pathways, most prominent of which is the induction of telomerase, leading to cell immortalization, such as in mammary epithelial cells. ${ }^{34}$ In breast cancer, BMI1 causes neoplastic transformation of lymphocytes and cooperates with H-Ras, giving rise to metastatic neoplasms in mice. A recent study has demonstrated that the activity of the estrogen receptor $\alpha$-coupled BMI1 signature impacts $\mathrm{p} 16^{\mathrm{INK} 4 \mathrm{a}}$ and cyclin D1 status, and correlates with the tumor molecular subtype and biologic behavior in breast cancer. ${ }^{11}$ Song et al overexpressed BMI1 in nasopharyngeal epithelial cells and found that the $\mathrm{pRb}$ pathway seemed to be specifically targeted by downregulation of $\mathrm{p} 16^{\mathrm{INK} 4 \mathrm{a}}$, resulting in cell immortalization. ${ }^{6}$ Although telomerase activity was induced in the process, BMI1-immortalized nasopharyngeal epithelial cells maintained a normal DNA damage response. ${ }^{6}$ Wang et al showed that downregulation of BMI1 could enhance cisplatin-sensitization by exacerbating reactive oxygen species production, leading to apoptosis through activation of the DNA damage response pathway. ${ }^{34}$ In addition, BMI1 is also highly expressed in CD133-positive liver CSCs, and 
BMI1 silencing completely abolishes the tumorigenicity. ${ }^{36,37}$ BMI1 has been found to be capable of inducing the epithelial mesenchymal transition, which is thought to promote invasion and metastasis of tumor cells. Additionally, lung cancer cellular models suggest that the epithelial mesenchymal transition can trigger conversion to a CSC phenotype. ${ }^{25,38}$

\section{BMII and EOC patients' stratification}

Because of its insidious onset, $\sim 70 \%$ of EOC patients are diagnosed at an advanced stage (International Federation of Gynecology and Obstetrics, [FIGO] III/IV stage) with a very poor prognosis. ${ }^{39}$ Lack of sensitive detection strategies to distinguish certain patients for more aggressive treatment remains a major obstacle in the management of EOC. The development and progression of this vexing carcinoma is presumed to be a multistep process involving multiple genetic and epigenetic changes. ${ }^{40}$ Thus, a substantial amount of research has focused on the discovery of specific biomarkers that are present in ovarian carcinoma cells, which could serve as reliable diagnostic and prognostic factors.

Ovarian cancer tissues express high levels of BMI1, and the expression intensity is significantly associated with the clinical parameters such as histological grade and phase of the disease by statistical analysis. ${ }^{33}$ Additionally, overexpression of BMI1 causes neoplastic transformation of lymphocytes in ovarian cancer. ${ }^{41}$ In accordance with this, our previous study demonstrated elevated expression of BMI1 in first-onset lymph node metastases, which was associated with shortened progression-free survival. ${ }^{42} \mathrm{Abd}$ El hafez and El-Hadaad tested BMI1 expression by immunohistochemistry method in paraffin-embedded tissues from 40 cases with EOC. ${ }^{19}$ And their results showed that BMI 1 was detected in $72.5 \%$ of the cases, of which $42.5 \%$ had high expression. Moreover, high BMI1 expression was strongly associated with advanced FIGO stages, a bilaterality pattern, higher Gynecologic Oncology Group grades, and serous histology $(P<0.05) .{ }^{19}$ Similarly, Yang et al found intensive expression of BMI 1 in $37 \%$ of ovarian carcinomas, $10 \%$ of borderline tumors, $3 \%$ of cystadenomas, and none of the normal ovaries, respectively. ${ }^{39}$ However, gene amplification of BMI1 was detected in merely $8 \%$ of ovarian carcinomas. Statistically significant associations were also found by Yang between intensive expression of BMI1 and the tumors ascending histological grade and FIGO stage. In addition, both studies demonstrated that BMI1 expression seemed to characterize a subset of patients with poor survival.

Collectively, these findings provide evidences that intensive expression of BMI1 might be important in the acquisition of an invasive and/or aggressive phenotype by ovarian carcinoma, and BMI1 could serve as an independent molecular marker for the identification of patients with a poor prognostic outcome (Table 1).

In addition, Glinsky et al applied a mouse/human comparative, translational genomics approach and identified a BMI1-driven 11-gene signature representing a stem cellresembling expression profile in prostate cancer. ${ }^{30}$ They further examined and validated the prognostic power of the 11-gene signature in several independent therapy-outcome sets in clinical samples obtained from 1,153 cancer patients diagnosed with eleven different types of cancer, including five epithelial malignancies (prostate, breast, lung, ovarian, and bladder cancers) and five nonepithelial malignancies (lymphoma, mesothelioma, medulloblastoma, glioma, and acute myeloid leukemia). The results suggest the presence of a conserved BMI1-driven pathway, which is engaged in a highly malignant subset of human cancers in a wide range of organs. The researchers assumed that instead of sole statistical analysis of cancer microarray data, including a relevant biological model in the signature discovery protocol

Table I Major studies performed about the clinical significance of BMII in EOC

\begin{tabular}{|c|c|c|c|}
\hline Study & Sample & Method & Clinical significance \\
\hline $\begin{array}{l}\text { Abd El hafez } \\
\text { and El-Hadaad }{ }^{19}\end{array}$ & 40 cancer tissues & $\mathrm{IHC}$ & $\begin{array}{l}\text { High BMII expression strongly associated with advanced FIGO stages, bilaterality, and } \\
\text { higher Gynecologic Oncology Group grades and carcinomas of serous histology. BMII } \\
\text { expression displayed a significant inverse association with OS. }\end{array}$ \\
\hline Zhang et $\mathrm{al}^{31}$ & 47 cancer tissues & $\mathrm{IHC}$ & $\begin{array}{l}\text { BMII expression levels in ovarian carcinoma tissue differ depending on tissue grade } \\
\text { (higher for G3 cancer cases than for grade G2 cases) and the stage of the disease } \\
\text { (lower for Phase II and III than for Phase IV cases). }\end{array}$ \\
\hline Yang et $\mathrm{al}^{39}$ & I 79 ovarian carcinomas & $\mathrm{IHC}, \mathrm{FISH}$ & $\begin{array}{l}\text { Significant positive associations were found between intensive expression of BMII } \\
\text { and the tumors ascending histological grade, later } \mathrm{pT} / \mathrm{pN} / \mathrm{pM} \text { and FIGO stages. BMII } \\
\text { expression was an independent prognostic factor for survival. }\end{array}$ \\
\hline Gui et $\mathrm{al}^{42}$ & $\begin{array}{l}100 \text { primary ovarian tumors; } \\
50 \text { LN and recurrent tumors }\end{array}$ & IHC, TMA & $\begin{array}{l}\text { BMII was heterogeneously expressed in primary versus recurrent tumors. Intensive } \\
\text { expression of BMII in the first-onset lymph node metastases and recurrent tumors was } \\
\text { associated with shortened PFS and shortened OS, respectively. }\end{array}$ \\
\hline
\end{tabular}

Abbreviations: BMII, B-cell-specific Moloney murine leukemia virus integration site I; EOC, epithelial ovarian cancer; FIGO, International Federation of Gynecologic Oncology; FISH, fluorescence in situ hybridization; IHC, immunohistochemistry; LN, lymph node; OS, overall survival; PFS, progression-free survival; TMA, tissue microarray. 
was more likely to identify outcomes common to multiple cancer types. ${ }^{43}$

\section{BMII and EOC treatment}

Although optimal cytoreductive surgery with platinum/taxol chemotherapy has markedly improved the prognosis for EOC patients, there are still patients who lack an initial response to frontline chemotherapy. Additionally, $\sim 70 \%$ of females with advanced ovarian cancers ultimately relapse and succumb to the devastating disease. Heterogeneity within individuals and tumors might affect therapeutic effectiveness, and treatment failure has often been attributed to chemoresistance. ${ }^{44}$ The frequently used clinicohistological parameters seem not to be representative of these refractory tumor cells, and accordingly are not suitable as indicators for the choice of subsequent treatment. As a consequence, more precise medicine is needed, and the underlying molecular events that control this tumor heterogeneity might provide us with potential targets for individualized treatment. Wang et al demonstrated that silencing BMI1 reduced intracellular glutathione levels and thereby sensitized chemoresistant ovarian cancer cells to antineoplastic medicines such as cisplatin. ${ }^{34}$ Furthermore, they knocked-down BMI1 by nanoliposomal delivery of small interfering RNA into an orthotopic mouse model of chemo-resistant ovarian cancer, and discovered that the combination of BMI1 silencing along with cisplatin almost completely abrogated ovarian tumor growth. Therefore, BMI1 might be an important new target for therapy in chemoresistant ovarian cancer.

Unfortunately, a specific mechanism for overexpression of BMI1 has not been determined. Yang et al found that the expression level of BMI1 protein in ovarian carcinoma did not always coincide with gene amplification, and our previous investigation revealed similar results in EOC tissue samples..$^{27,39,42}$ Indeed, in bladder cancers, a significant difference in BMI1 protein expression and messengerRNA (mRNA) levels was obtained, but BMI1 protein was upregulated to a much greater extent than BMI1 mRNA in cancerous tissue compared to noncancerous tissues, implying that the major source of BMI1 expression might be dysregulated at the posttranscriptional level. ${ }^{45}$ However the transcriptional and posttranscriptional regulation of BMI1 in human malignancies remains largely elusive. Bhattacharyya et al found that BMI1 expression was increased during disease progression in patients with chronic myeloid leukemia, as well as the discrepancy between the amount of protein and mRNA. They intended to elucidate the underlying molecular mechanism by transduction of $B C R-A B L$ vector to chronic myeloid leukemia cell lines. And their findings suggested the possibility that an additional genetic alteration might exert a positive effect on the stabilization of BMI1 protein, providing an upstream signal to regulate BMI1 expression. ${ }^{46}$

Epigenetics is defined as heritable changes in gene expression that do not alter the DNA sequence itself. The mechanisms responsible for such changes include DNA methylation, histone modification, and micro RNA (miRNA), etc. Emerging evidence suggests that altered regulation of miRNA is involved in the pathogenesis of many cancers and miR-15a and miR-16 were identified as underexpressed in ovarian cell lines and primary ovarian tissues. ${ }^{33,47}$ Bhattacharya et al showed that miRNAs directly target the BMI1 $3^{\prime}$ untranslated region and significantly downregulates BMI1 protein levels, which led to significant reduction in ovarian cancer cell proliferation and clonal growth. ${ }^{33}$ These findings suggest the potential of therapeutic strategies that restore miRNAs (eg, miR-15a and miR-16) expression in ovarian cancer and in other cancers that involve upregulation of BMI1.

\section{Future challenges}

A large amount of research has demonstrated that intense expression of BMI1 was a predictor of poor survival outcomes in various human malignancies, including ovarian cancer. Studies in literatures have conflicting results regarding the prognostic value of the $\mathrm{PcG}$ proteins. In breast cancer, overexpression of BMI1 was reported to have different influences on patient prognosis by Pietersen et al and BMI1 was found to have no prognostic value in urothelial carcinoma of the bladder. ${ }^{45,48}$ In contrast, survival data from Benard et al also showed that high expression of BMI1 was associated with better patient survival and longer recurrence-free periods, compared to prostate cancer patients with low BMI1 expression. ${ }^{10}$ Single-institution-based research with limited sample sizes might account for these inconsistent findings, while tissue/organ specificity of oncogenetic BMI1 activation needs further investigation as a priority. Additionally, the relationship between BMI1 and clinicopathological variables in EOC should be validated at both the genetic and epigenetic level by multiple centers and with large case volumes.

The BMI1-driven 11-gene signature protocol established by Glinsky et al inspired us to think that the combined measurement and analysis of relevant biomarkers might be a more reliable strategy for risk assessment. ${ }^{12,30}$ Similarly, Benard et al showed that high expression of all four markers (BMI1, EZH2, SUZ12, and H3K27me3) provided a more pronounced hazard ratio and effect on survival outcomes, 
compared to evaluation with each individual marker. ${ }^{10}$ The results of these combined marker analyses underline the cooperation of these three enzymes in PcG complexes, and thus provide a better risk stratification of patients. In addition, the major functionality of PcG proteins itself, histone modifications, could serve as valuable epigenetic markers for clinical application. In ovarian cancer, decreased expression of polycomb repressive complexmediated $\mathrm{H} 3 \mathrm{~K} 27 \mathrm{me} 3$ is significantly associated with high grade and advanced stage, and can predict resistance to chemotherapy as well. ${ }^{49}$ However, the relationship between BMI1-participant H2AK119ub1 and clinical outcome in EOC is unclear. Modern and mature sequencing technology makes multiple-molecular test pattern practical for genetic, transcriptional, and post-transcriptional analyses. These comprehensive results will further unravel the underlying molecular image for each individual tumor and advance the search for new biomarkers to be used in a clinical setting, so as to achieve precision treatment eventually.

The development and progression of EOC may involve the accumulation of multiple genetic alterations over a long period of time. However, the molecular basis of this carcinoma is far from being fully understood. Tumor heterogeneity and CSCs have characteristic molecular traits. Definition of these subtypes will allow treatment to be tailored directly for each type of EOC, and marked progress can be expected in improving patient survival rate. However, resistance to chemotherapy and varying responses to specific drugs, which may be found de novo or may be acquired, still present a therapeutic challenge. Although the role of BMI1 and the related network has been studied extensively in recent years, data regarding BMI1 in tumor biology is far from complete. Especially, a specific mechanism for its dysregulation in cancers has not been determined..$^{50}$ Therefore, much effort is still needed to characterize the complete molecular details, in order to lay the foundation for transition medicine from the experimental findings to potential clinical applications in EOC.

\section{Conclusion}

The currently used clinical variables (eg, histological type, FIGO stage) lack efficacy and accuracy to stratify patients based on distinct therapeutic responses, leaving refractory and recurrent diseases a vexing challenge in the management of EOC. The molecular traits underlying CSCs and tumor heterogeneity have provided us with a new perspective. Aberrant expression of BMI1, a stem-cell marker and PcG member, has been validated to correlate with clinical characteristics and survival outcomes in a diverse set of human malignancies, including EOC. However, the biological and oncogenetic events related to BMI1 still need further comprehensive investigations to qualify BMI1 as a reliable molecular marker for precise patients' stratification and therapeutic targeting of EOC.

\section{Disclosure}

The authors report no conflicts of interest in this work.

\section{References}

1. Cooke SL, Brenton JD. Evolution of platinum resistance in high-grade serous ovarian cancer. Lancet Oncol. 2011;12(12):1169-1174.

2. van Lohuizen M, Verbeek S, Scheijen B, Wientjens E, van der Gulden H, Berns A. Identification of cooperating oncogenes in E mu-myc transgenic mice by provirus tagging. Cell. 1991;65:737-752.

3. Huber GF, Albinger-Hegyi A, Soltermann A, et al. Expression patterns of Bmi-1 and p16 significantly correlate with overall, disease-specific, and recurrence-free survival in oropharyngeal squamous cell carcinoma. Cancer. 2011;117(20):4659-4670.

4. Siddique HR, Saleem M. Role of BMI1, a stem cell factor, in cancer recurrence and chemoresistance: preclinical and clinical evidences. Stem Cells. 2012;30(3):372-378.

5. Cao L, Bombard J, Cintron K, Sheedy J, Weetall ML, Davis TW. BMI1 as a novel target for drug discovery in cancer. J Cell Biochem. 2011; 112(10):2729-2741.

6. Song LB, Zeng MS, Liao WT, et al. Bmi-1 is a novel molecular marker of nasopharyngeal carcinoma progression and immortalizes primary human nasopharyngeal epithelial cells. Cancer Res. 2006;66(12): 6225-6232.

7. Huber GF, Albinger-Hegyi A, Soltermann A, et al. Expression patterns of Bmi-1 and p16 significantly correlate with overall, disease-specific, and recurrencefree survival in oropharyngeal squamous cell carcinoma. Cancer. 2011;117(20):4659-4670.

8. Marquardt JU, Factor VM, Thorgeirsson SS. Epigenetic regulation of cancer stem cells in liver cancer: current concepts and clinical implications. J Hepatol. 2010;53(3):568-577.

9. Crea F, Serrat MAD, Hurt EM, Thomas SB, Danesi R, Farrar WL. BMI1 silencing enhances docetaxel activity and impairs antioxidant response in prostate cancer. Int J Cancer. 2011;128:1946-1954.

10. Benard A, Goossens-Beumer IJ, van Hoesel AQ, et al. Prognostic value of polycomb proteins EZH2, BMI1 and SUZ12 and histone modification H3K27me3 in colorectal cancer. PLoS One. 2014;9(9):e108265.

11. Wang H, Liu H, Li X, et al. Estrogen receptor $\alpha$-coupled Bmi1 regulation pathway in breast cancer and its clinical implications. $B M C$ Cancer. 2014;14:122.

12. Park IK, Morrison SJ, Clarke MF. Bmi1, stem cells, and senescence regulation. J Clin Invest. 2004;113(2):175-179.

13. Engelsen IB, Mannelqvist M, Stefansson IM, et al. Low BMI-1 expression is associated with an activated BMI-1-driven signature, vascular invasion, and hormone receptor loss in endometrial carcinoma. Br J Cancer. 2008;98(10):1662-1669.

14. Allegra E, Puzzo L, Zuccalà V, et al. Nuclear BMI-1 expression in laryngeal carcinoma correlates with lymph node pathological status. World J Surg Oncol. 2012;10:206.

15. Francis NJ, Kingston RE, Woodcock CL. Chromatin compaction by a polycomb group protein complex. Science. 2004;306(5701): 1574-1577.

16. Zhou W, Zhu P, Wang J, et al. Histone H2A monoubiquitination represses transcription by inhibiting RNA polymerase II transcriptional elongation. Mol Cell. 2008;29(1):69-80.

17. Morey L, Helin K. Polycomb group protein-mediated repression of transcription. Trends Biochem Sci. 2010;35(6):323-332. 
18. Park IK, Qian D, Kiel M, et al. Bmi-1 is required for maintenance of adult self-renewing haematopoietic stem cells. Nature. 2003; 423(6937):302-305.

19. Abd El hafez A, El-Hadaad HA. Immunohistochemical expression and prognostic relevance of Bmi-1, a stem cell factor, in epithelial ovarian cancer. Ann Diagn Pathol. 2014;18(2):58-62.

20. Lessard J, Schumacher A, Thorsteinsdottir U, van Lohuizen M, Magnuson T, Sauvageau G. Functional antagonism of the PolycombGroup genes eed and BMI1 in hemopoietic cell proliferation. Genes Dev. 1999;13(20):2691-2703.

21. Leung C, Lingbeek M, Shakhova O, et al. BMI1 is essential for cerebellar development and is overexpressed in human medulloblastomas. Nature. 2004;428:337-341.

22. Molofsky AV, He S, Bydon M, Morrison SJ, Pardal R. BMI1 promotes neural stem cell self-renewal and neural development but not mouse growth and survival by repressing the p16Ink4a and p19Arf senescence pathways. Genes Dev. 2005;19:1432-1437.

23. Zacharek SJ, Fillmore CM, Lau AN, et al. Lung stem cell self-renewal relies on BMI1-dependent control of expression at imprinted loci. Cell Stem Cell. 2011;9(3):272-281.

24. Malouf GG, Taube JH, Lu Y, et al. Architecture of epigenetic reprogramming following Twist1 mediated epithelial mesenchymal transition. Genome Biol. 2013;14(12):R144.

25. Heffner M, Fearon DT. Loss of T cell receptor-induced Bmi-1 in the KLRG1(+) senescent CD8(+) T lymphocyte. Proc Natl Acad Sci US A. 2007;104(33):13414-13419.

26. Pethe P, Pursani V, Bhartiya D. Lineage specific expression of Polycomb Group Proteins in human embryonic stem cells in vitro. Cell Biol Int. 2015;39(5):600-610.

27. Zeng J, Ruan J, Luo L, et al. Molecular portraits of heterogeneity related to cancer stem cells in human ovarian cancer. Int J Gynecol Cancer. 2014;24(1):29-35.

28. Pardal R, Clarke MF, Morrison SJ. Applying the principles of stem-cell biology to cancer. Nat Rev Cancer. 2003;3:895-902.

29. Alamodi AA, Eshaq AM, Hassan SY, et al. Cancer stem cell as therapeutic target for melanoma treatment. Histol Histopathol. 2016; $15: 11791$.

30. Glinsky GV, Berezovska O, Glinskii AB. Microarray analysis identifies a death-from-cancer signature predicting therapy failure in patients with multiple types of cancer. J Clin Invest. 2005;115(6): 1503-1521.

31. Zhang F, Sui L, Xin T. Correlations of Bmi-1 expression and telomerase activity in ovarian cancer tissues. Exp Oncol. 2008;30(1):70-74.

32. Allegra E, Trapasso S, Pisani D, Puzzo L. The role of BMI1 as a biomarker of cancer stem cells in head and neck cancer: a review. Oncology. 2014;86(4):199-205.

33. Bhattacharya R, Nicoloso M, Arvizo R, et al. MiR-15a and MiR-16 control Bmi-1 expression in ovarian cancer. Cancer Res. 2009;69(23): 9090-9095.
34. Wang E, Bhattacharyya S, Szabolcs A, et al. Enhancing chemotherapy response with Bmi-1 silencing in ovarian cancer. PLoS One. 2011; 6(3):e17918.

35. Valk-Lingbeek ME, Bruggeman SW, Van LM. Stem cells and cancer; the polycomb connection. Cell. 2004;118(4):409-418.

36. Chiba T, Zheng YW, Kita K, et al. Enhanced self-renewal capability in hepatic stem/progenitor cells drives cancer initiation. Gastroenterology. 2007;133(3):937-950.

37. Ma S, Chan KW, Hu L, et al. Identification and characterization of tumorigenic liver cancer stem/progenitor cells. Gastroenterology. 2007; 132:2542-2556.

38. Koren A, Rijavec M1, Kern I, Sodja E, Korosec P, Cufer T. BMI1, ALDH1A1, and CD133 transcripts connect epithelial-mesenchymal transition to cancer stem cells in lung carcinoma. Stem Cells Int. 2016; 2016:9714315.

39. Yang GF, He WP, Cai MY, et al. Intensive expression of Bmi-1 is a new independent predictor of poor outcome in patients with ovarian carcinoma. BMC Cancer. 2010;10:133.

40. Disaia PJ, Bloss JD. Treatment of ovarian cancer: new strategies. Gynecol Oncol. 2003;90(2):S24-S32.

41. Bhattacharya R, Nicoloso M, Arvizo R, et al. MiR-15a and MiR-16 control Bmi-1 expression in ovarian cancer. Cancer Res. 2009;69(23): 9090-9095.

42. Gui T, Bai H, Zeng J, et al. Tumor heterogeneity in the recurrence of epithelial ovarian cancer demonstrated by polycomb group proteins. Onco Targets Ther. 2014;7:1705-1716.

43. Rhodes DR, Yu J, Shanker K, et al. Large-scale meta-analysis of cancer microarray data identifies common transcriptional profiles of neoplastic transformation and progression. Proc Natl Acad Sci U S A. 2004; 101(25):9309-9314.

44. Siegel RL, Miller KD, Jemal A. Cancer statistics, 2015. CA Cancer J Clin. 2015;65(1):5-29.

45. Qin ZK, Yang JA, Ye YL, et al. Expression of Bmi-1 is a prognostic marker in bladder cancer. BMC Cancer. 2009;9:61.

46. Bhattacharyya J, Mihara K, Yasunaga S, et al. BMI-1 expression is enhanced through transcriptional and posttranscriptional regulation during the progression of chronic myeloid leukemia. Ann Hematol. 2009;88(4):333-340.

47. Bartel DP. MicroRNAs: genomics, biogenesis, mechanism, and function. Cell. 2004;116(2):281-297.

48. Pietersen AM, Horlings HM, Hauptmann M, et al. EZH2 and BMI1 inversely correlate with prognosis and TP53 mutation in breast cancer. Breast Cancer Res. 2008;10(6):R109.

49. Bai H, Cao D, Yang J, Li M, Zhang Z, Shen K. Genetic and epigenetic heterogeneity of epithelial ovarian cancer and the clinical implications for molecular targeted therapy. J Cell Mol Med. 2016;20(4):581-593.

50. Gialmanidis IP, Bravou V, Petrou I, et al. Expression of Bmi1, FoxF1, nanog, and gamma-catenin in relation to hedgehog signaling pathway in human non-small-cell lung cancer. Lung. 2013;191(5):511-521.
OncoTargets and Therapy

\section{Publish your work in this journal}

OncoTargets and Therapy is an international, peer-reviewed, open access journal focusing on the pathological basis of all cancers, potential targets for therapy and treatment protocols employed to improve the management of cancer patients. The journal also focuses on the impact of management programs and new therapeutic agents and protocols on
Dovepress

patient perspectives such as quality of life, adherence and satisfaction. The manuscript management system is completely online and includes a very quick and fair peer-review system, which is all easy to use. Visit http://www.dovepress.com/testimonials.php to read real quotes from published authors. 